\section{Metabolic Profiling in Pharmaceutical Drug Discovery - Robust and Automated Analysis of Metabonomic Data Sets}

Götz Schlotterbeck ${ }^{\star a}$, Frank Dieterle ${ }^{b}$, Alfred Ross ${ }^{\mathrm{a}}$, and Hans Senna

*Correspondence: Dr. G. Schlotterbecka , Tel.: +4161 68807 52, Fax: +4161688 74 08, E-Mail: goetz.schlotterbeck@roche.com

F. Hoffmann-La Roche, Pharmaceutical Division, PRBD-E, CH-4070 Basel

${ }^{b}$ Novartis Pharma AG, Exploratory Development, $\mathrm{CH}-4002$ Basel

Keywords: Biofluid · Mass spectrometry · Metabolic profiling · Metabolite projection analysis · Metabolomics · Metabonomics . NMR spectroscopy

Metabolic profiling of biofluids or tissues, also known as metabonomics or metabolomics is a platform to investigate the metabolic state of a biological system. Metabonomics is applied in nutrition, agricultural research and more widely in pharmaceutical preclinical and clinical settings for safety assessment of drug candidates, e.g. relating to nephrotoxicity, hepatotoxicity, phospholipidosis or peroxisome proliferation.

Metabonomics involves the determination of changes in the concentration levels of low molecular weight endogenous metabolites in biological samples resulting from physiological stimuli or genetic modification. The aim of metabolic profiling is to extract latent biochemical information that is of diagnostic and prognostic value and which reflects 'actual' biological events in the network of metabolic pathways, as opposed to the potential for such events reflected by DNA or mRNA. Thus metabolomics provides a complementary aspect and useful connection between other '-omics' platforms (transcriptomics/proteomics) and the actual metabolic state and tissue histology.

The analytical technologies typically include nuclear magnetic resonance (NMR) and mass spectroscopy (MS) in combination with a separation method such as gas or liquid chromatography (GC, LC). NMR and MS complement each other in capturing metabolic data, both in terms of sensitivity, ability for quantification, and potential for structure elucidation of unknowns. Due to the complexity of spectra of biofluids or tissues, statistical and multivariate data analysis tools are used to identify and quantify significant changes of metabolites.

Recently, we introduced metabolite projection analysis (MPA), a fast and automated way supporting identification of significantly changed metabolites in a set of NMR spectra taken on body fluids of treated and control group individuals.

Fast and robust preprocessing of complex metabolic profiling data for multivariate data analysis can now be achieved. Significantly changed metabolites in metabonomics data sets can be easily identified and assigned to metabolites without the need to inspect a high number of NMR spectra. Thus metabonomics data sets can now be analyzed easier, faster, more reliable and more accurate.

Received: July 31, 2007

\section{References}

F. Dieterle, A. Ross, G. Schlotterbeck, H. Senn, Anal. Chem. 2006, 78, 3551.

G. Schlotterbeck, A. Ross, F. Dieterle, H. Senn, Pharmacogenomics 2006, 7, 1055.

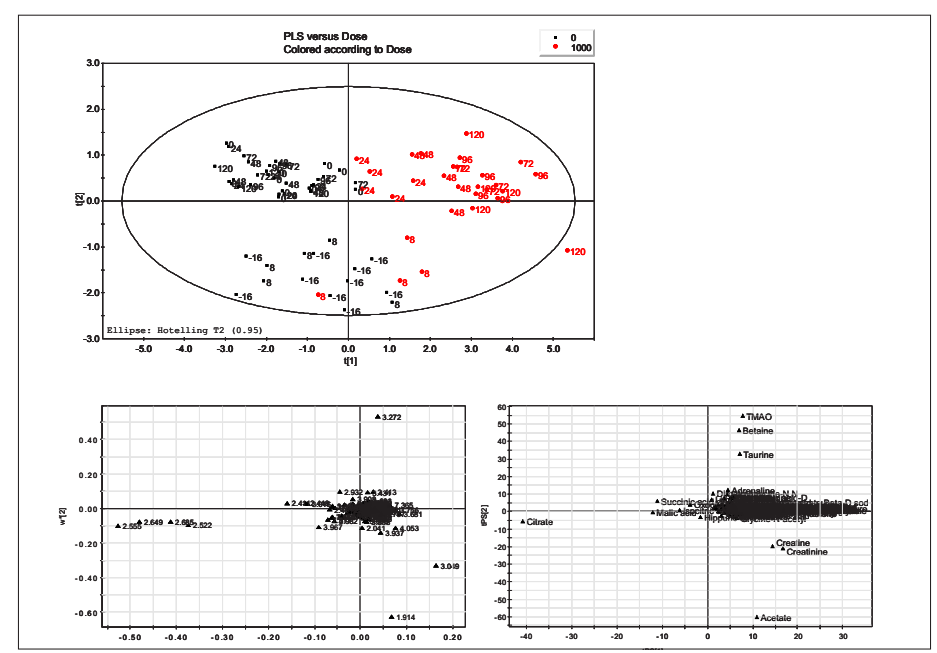

Score plot of a Projection to Latent Structures (PLS) model of rat urine samples colored by dose level (top). In the standard weights plot (bottom left), the class-separating variables are labeled with chemical shifts. The corresponding score plot of MPA is shown at the bottom right. Here, the read out are significantly changed metabolites rather than only chemical shifts.
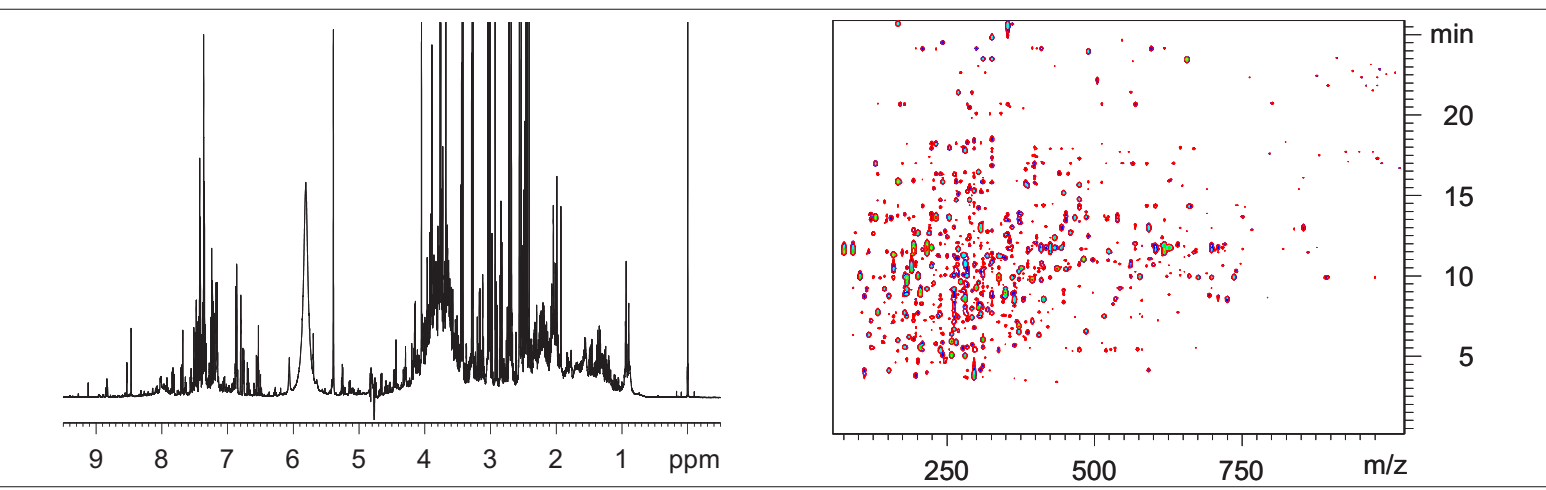

${ }^{1} \mathrm{H}$ NMR spectrum of rat urine recorded at $600 \mathrm{MHz}$ (left) and LC-MS profile (positive mode) of rat urine (right) 